\title{
Relationship between Helium Degassing of Cattle-Manure-Compost Adsorbents and Copper Ions Removal
}

\author{
Muhammad Abbas Ahmad Zaini ${ }^{1}$, Mohd Azizi Che Yunus ${ }^{1}$, Yoshimasa Amano ${ }^{2}$, Motoi Machida ${ }^{2}$ \\ ${ }^{1}$ Centre of Lipids Engineering \& Applied Research, Universiti Teknologi Malaysia Skudai, Johor, Malaysia \\ ${ }^{2}$ Safety and Health Organization Chiba University, Chiba, Japan \\ Email: abbas@cheme.utm.my, machida@faculty.chiba-u.jp
}

Received May 5, 2012; revised June 17, 2012; accepted June 25, 2012

\begin{abstract}
This work was aimed to investigate the effect of helium degassing of cattle-manure-compost (CMC) based activated carbons on the adsorptive removal of copper ions from aqueous solution. Degassing temperatures were $500^{\circ} \mathrm{C}, 800^{\circ} \mathrm{C}$ and $1000^{\circ} \mathrm{C}$. Activated carbons were characterized according to surface chemistry and pore structures. Adsorption of copper ions was carried out using the conventional bottle-point technique to which the equilibrium data were correlated to Langmuir and Freundlich models. Results indicated that the uptake of copper ions could be well characterized by Langmuir model. It was found that the adsorption of copper ions decreased with significant decrease in surface area as a result of helium degassing at higher temperature. The increase of electron density on graphene layers offered higher affinity towards copper ions at lower equilibrium concentration. It was inferred that copper ions favorably adsorbed on mesopores at lower equilibrium concentration and switched to micropores at higher equilibrium concentration.
\end{abstract}

Keywords: Activated Carbon; Adsorption; Copper Ions; Cattle-Manure-Compost; Helium Degassing

\section{Introduction}

Heavy metals are toxic, not biodegradable and can easily enter the food chain because of high solubility in water. Excessive consumption of metal ions-contaminated water can cause acute illnesses such as diarrhea, nausea, brain disorders, liver and renal dysfunctions, and cancers [1].

Adsorption is a preferred choice amongst other physico-chemical techniques of heavy metals remediation [2]. It can be described as a mass transfer process by which a desired substance is transferred from the liquid phase to the surface of a solid adsorbent, and becomes bound by a physical or chemical attraction [3]. The most widely used adsorbent is activated carbon, a complex and heterogeneous material with unique adsorption characteristics. Adsorption of metal ions onto activated carbon is commonly influenced by surface area, pore volume and surface functionalities.

However, commercial activated carbon suffers from a number of drawbacks. The precursors like coal and petroleum pitch are not renewable, while regeneration of spent activated carbon is relatively expensive. Global demand of activated carbon is forecasted to be $5 \%-10 \%$ a year [4], and steep increase of price would also be anticipated.
This recent scenario has brought about searches for new alternatives that are abundantly available and low cost [5]. An interesting material under this category is cattle-manure-compost (CMC) [6], a residue of temperature-phased anaerobic digestion for methane generation. Such waste can become a source of pollution and threat to public health if they are not properly handled and disposed. Therefore, converting CMC into activated carbon is a good strategy to overcome the aforesaid problems and could also be used to mitigate other pollutions [7].

Conversion of CMC into activated carbon was first described by Qian and co-workers [6]. It was reported that the nitrogen content is nearly 6 times higher than that of commercial Filtrasorb 400 (F400, Calgon Mitsubishi Chemical Corporation) [8]. Our previous work [8] suggested that the selective removal of copper ions over lead ions was partly due to the nitrogen-rich surface of $\mathrm{CMC}$-activated carbons. The present work was aimed to investigate the effect of helium degassing of CMC-activated carbons on adsorptive removal of copper ions.

\section{Materials and Methods}

\subsection{Preparation of Activated Carbon}

Cattle-manure-compost (CMC) was obtained from JFE 
Corporation, Japan. Pre-treatment steps were described in detail elsewhere [6]. $\mathrm{ZnCl}_{2}$, the activating agent, was dissolved and impregnated into $\mathrm{CMC}$ at weight ratios of 0.5 and 1.5 . One-step activation was progressed at $500^{\circ} \mathrm{C}$ for $1 \mathrm{~h}$ under the flow of $\mathrm{N}_{2}$. The resultant products were then treated in $3 \mathrm{M}$ hydrochloric acid and concentrated hydrofluoric acid. Thereafter, they were washed with de-ionized water until the solution $\mathrm{pH}$ became constant. Degassing procedure was progressed at temperatures of 500,800 and $1000^{\circ} \mathrm{C}$ under the flow of high purity helium gas for $1 \mathrm{~h}$. The activated carbons were designated as CxTy where $\mathrm{C}$ is activated carbon with $\mathrm{x}$ referring to impregnation ratio, while $\mathrm{T}$ represents helium degassing at y temperature. Thus, $\mathrm{C} 1 \mathrm{~T} 5$ refers to impregnation ratio of 0.5 and degassing temperature of $500^{\circ} \mathrm{C}$, while $\mathrm{C} 2 \mathrm{~T} 10$ indicates impregnation ratio of 1.5 and $1000^{\circ} \mathrm{C}$ degassing temperature.

\subsection{Characterization of Activated Carbon}

Surface functionalities were estimated using methods described by Boehm [9]. Batches of $300 \mathrm{mg}$ carbon were brought into contact with $15 \mathrm{~mL}$ solutions of $0.1 \mathrm{M} \mathrm{Na}$ $\mathrm{HCO}_{3}, 0.05 \mathrm{M} \mathrm{Na}_{2} \mathrm{CO}_{3}, 0.1 \mathrm{M} \mathrm{NaOH}$ and $0.1 \mathrm{M} \mathrm{HCl}$, and were allowed to equilibrate at $25^{\circ} \mathrm{C}$ for $48 \mathrm{hrs}$. The aliquots were back-titrated with either $0.05 \mathrm{M} \mathrm{HCl}$ for surface acidic functional groups or $0.1 \mathrm{M} \mathrm{NaOH}$ for basic groups, at which the neutralization points were observed using universal $\mathrm{pH}$ indicators.

Batches of $100 \mathrm{mg}$ carbon were brought into contact with $50 \mathrm{ml}$ of $0.1 \mathrm{M} \mathrm{NaCl}$ at different initial $\mathrm{pH}$, and were allowed to equilibrate at $25^{\circ} \mathrm{C}$ for $24 \mathrm{hrs}$. The value of $\mathrm{pH}_{\mathrm{PZC}}$ was determined when the equilibrium $\mathrm{pH}$ is equal to the initial $\mathrm{pH}[10]$.

Pore characteristics were determined using Beckman Coulter SA3100 surface area analyzer (USA) at $77 \mathrm{~K}$, while elemental compositions were measured using a Perkin-Elmer PE2400 microanalyzer.

\subsection{Adsorption Studies}

Analytical-reagent grade chemicals were purchased from Kanto Chemical Co., Inc. Stock solutions of model metal ions-contaminated water were prepared by dissolving desired weight of $\mathrm{CuCl}_{2} \cdot 2 \mathrm{H}_{2} \mathrm{O}$ in de-ionized water. Adsorption of metal ions was carried out at $25^{\circ} \mathrm{C}$ for $48 \mathrm{~h}$ in a water bath orbital shaker. Fixed amount of activated carbon was added to the conical flasks containing $50 \mathrm{ml}$ of simulated wastewater with known concentration. The values of initial solution $\mathrm{pH}$ were measured as $5.2 \pm 0.3$. The amount of metal ions adsorbed, $q_{e}(\mathrm{mmol} / \mathrm{g})$ was calculated as $q_{e}=\left(C_{0}-C_{e}\right) \times(V / m)$, where $C_{0}$ and $C_{e}$ are respectively the initial and equilibrium concentrations in $\mathrm{mmol} / \mathrm{L}, V(\mathrm{~L})$ is the volume of solution and $m(\mathrm{~g})$ is the mass of carbon. Concentration of copper ions was meas- ured using atomic absorption spectroscopy (Rigaku novAA 300).

\section{Results and Discussion}

\subsection{Characteristics of Activated Carbon}

Results of Boehm titration and elemental analysis are shown in Table 1. At $500^{\circ} \mathrm{C}$ of helium degassing, carboxylic groups of $\mathrm{CMC}$-activated carbons were readily liberated and acidic groups were dominated by phenolic groups.

The concentration of acidic groups gradually decreased with the increase of degassing temperature to $1000^{\circ} \mathrm{C}$. The surface of activated carbons became more basic as the degassing temperature increases. This was well tallied with the values of $\mathrm{pH}_{\text {PzC }}$. In the absence of acidic groups, basic groups are mainly attributed to electron-donating character and delocalized $\pi$-electrons on graphene layers that could behave as Lewis bases.

The increase of degassing temperature also results in the decrease of nitrogen content to about $65 \%$. The leftover nitrogen moieties may consist of quaternary nitrogen, pyridinic and pyrrolic, while the fate of nitrogen functional groups in the non-cyclized structure will be the same as that of acidic functional groups. In general, both $\mathrm{C} 1$ and $\mathrm{C} 2$ activated carbons series show identical trend of chemical properties with respect to helium degassing temperatures.

Table 2 displays the physical characteristics of CMCactivated carbons. Slight decrease in yield implies the

Table 1. Chemical properties of activated carbons.

\begin{tabular}{cccccc}
\hline \multirow{2}{*}{ Carbon } & $\mathbf{p H}_{\mathbf{p z c}}$ & \multicolumn{2}{c}{$\begin{array}{c}\text { Functional } \\
\text { Groups (mmol/g) }\end{array}$} & \multicolumn{2}{c}{$\begin{array}{c}\text { Elemental } \\
\text { Composition (\%) }\end{array}$} \\
\cline { 3 - 6 } & & Total acidic & Basic & Carbon & Nitrogen \\
\hline C1T5 & 7.7 & 0.71 & 0.47 & 85.9 & 2.03 \\
C1T8 & 10.2 & 0.49 & 0.59 & 92.8 & 1.59 \\
C1T10 & 10.4 & 0.29 & 0.74 & 91.2 & 0.65 \\
C2T5 & 8.5 & 0.78 & 0.47 & 90.4 & 1.82 \\
C2T8 & 10.3 & 0.52 & 0.60 & 94.6 & 1.38 \\
C2T10 & 10.4 & 0.28 & 0.67 & 93.5 & 0.70 \\
\hline
\end{tabular}

Table 2. Yield and pore characteristics.

\begin{tabular}{cccccc}
\hline \multirow{2}{*}{ Carbon } & Yield (\%) & \multicolumn{4}{c}{ Pore Characteristics } \\
\cline { 3 - 6 } & & $\begin{array}{c}{ }^{\mathrm{a}} \boldsymbol{S}_{\text {BET }} \\
\left(\mathbf{m}^{\mathbf{2}} \mathbf{g}\right)\end{array}$ & $\begin{array}{c}{ }^{\mathrm{b}} \boldsymbol{V}_{\text {total }} \\
(\mathbf{m l} / \mathbf{g})\end{array}$ & $\begin{array}{c}{ }^{\mathrm{c}} \boldsymbol{R}_{\text {meso }} \\
\mathbf{( \% )}\end{array}$ & $\begin{array}{c}{ }^{\mathrm{d}} \boldsymbol{D}_{\text {average }} \\
\mathbf{( n m )}\end{array}$ \\
\hline C1T5 & 39.2 & 961 & 0.51 & 27 & 2.10 \\
C1T8 & 36.2 & 702 & 0.38 & 25 & 2.16 \\
C1T10 & 35.6 & 704 & 0.39 & 28 & 2.19 \\
C2T5 & 39.7 & 1779 & 1.06 & 77 & 2.39 \\
C2T8 & 36.6 & 1220 & 0.70 & 68 & 2.30 \\
C2T10 & 36.4 & 1182 & 0.68 & 66 & 2.29 \\
\hline
\end{tabular}

a. BET surface area; b. Total pore volume; c. Mesopore content; d. Average pore width. 
effectiveness of helium degassing to remove surface functional groups at high temperature. The higher the amount of $\mathrm{ZnCl}_{2}$ used for activation, the more intense its dehydrating effect to enlarge the surface area so as to increase the mesopore content [6]. And as a result, the surface area and mesopore content of $\mathrm{C} 2$ was greater than those of $\mathrm{C} 1$. Both $\mathrm{C} 1$ and $\mathrm{C} 2$ series showed a decrease in surface area with the increase of degassing temperature. Yet, the decrease of $\mathrm{C} 2$ series was more prevalent because it was originally rich in mesopore structure that is prone to diminish at higher temperature. Average pore widths were within the lower edge of mesopores ranging from $2.10 \mathrm{~nm}$ to $2.39 \mathrm{~nm}$.

\subsection{Adsorption of Copper Ions}

Uptake of copper ions by $\mathrm{C} 1$ series is illustrated in Figure 1. At equilibrium concentration below $0.3 \mathrm{mmol} / \mathrm{L}$, C1T10 showed a superior performance as compared to C1T8 and C1T5. As the equilibrium concentration increases, the uptake by all $\mathrm{C} 1$-activated carbons began to leveled-off at about $0.55 \mathrm{mmol} / \mathrm{g}$. Slight decrease in surface area of $\mathrm{C} 1$ series upon helium degassing did not affect the saturation point towards copper ions removal. It was also found that the uptake mechanism was not affected by the decrease of acidic groups.

Figure 2 shows the uptake of copper ions by $\mathrm{C} 2$ series. The adsorption was obviously driven by surface area, because the higher the surface area the greater the interaction probabilities to accommodate copper ions. A significant decrease of $600 \mathrm{~m}^{2} / \mathrm{g}$ in surface area upon helium degassing decreases the removal of copper ions at higher equilibrium concentration. Although C2T10 showed a greater surface area than $\mathrm{C} 1 \mathrm{~T} 10$, the saturation point of C2T10 was slightly lower as compared to C1T10. It is suggested that copper ions are preferably dehydrated on the micropore channels at higher equilibrium concentration [8].

The increase of equilibrium $\mathrm{pH}$ in adsorption with increasing temperature of degassing of activated carbons indicates that the surface has been protonated and becomes positively charged. This occurs whenever the equilibrium $\mathrm{pH}$ is lesser than $\mathrm{pH}_{\mathrm{PZC}}$, and commonly results in a lower uptake of metal ions due to repulsive force from the surface. Because of the surface is no longer plentiful in acidic groups as the degassing temperature increases, attempt to increase solution $\mathrm{pH}$ above $\mathrm{pH}_{\mathrm{PZC}}$ in order to instigate negatively charged surface will offer trivial outcomes. Moreover, adjusting the $\mathrm{pH}$ to basic for copper-bearing solution will give better chance for precipitate complex to evolve [8].

Two generally used isotherm models, namely Langmuir and Freundlich, were employed to characterize the adsorption data. The Langmuir model assumes uniform monolayer adsorption on a finite number of identical and

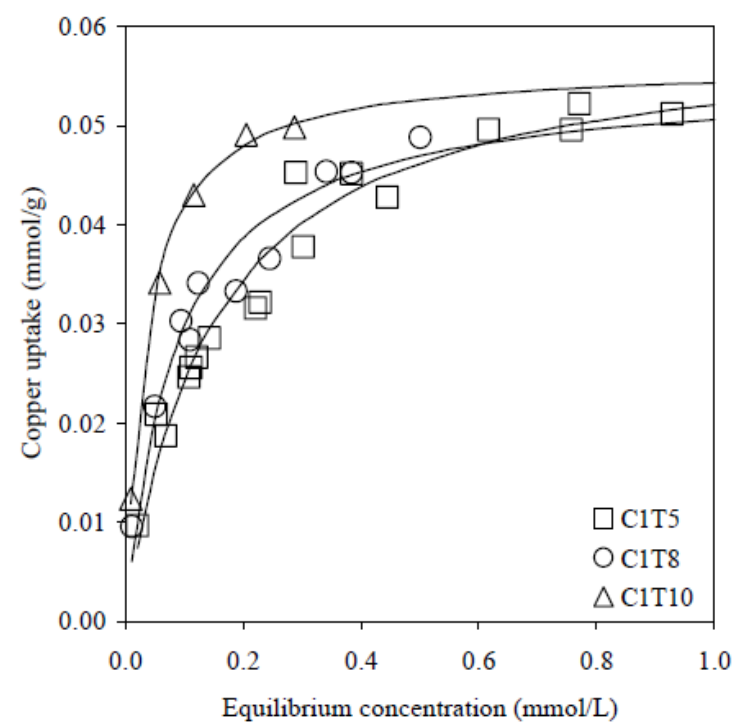

Figure 1. Adsorption of copper ions by C1 activated carbons series. Lines were predicted from Langmuir model.

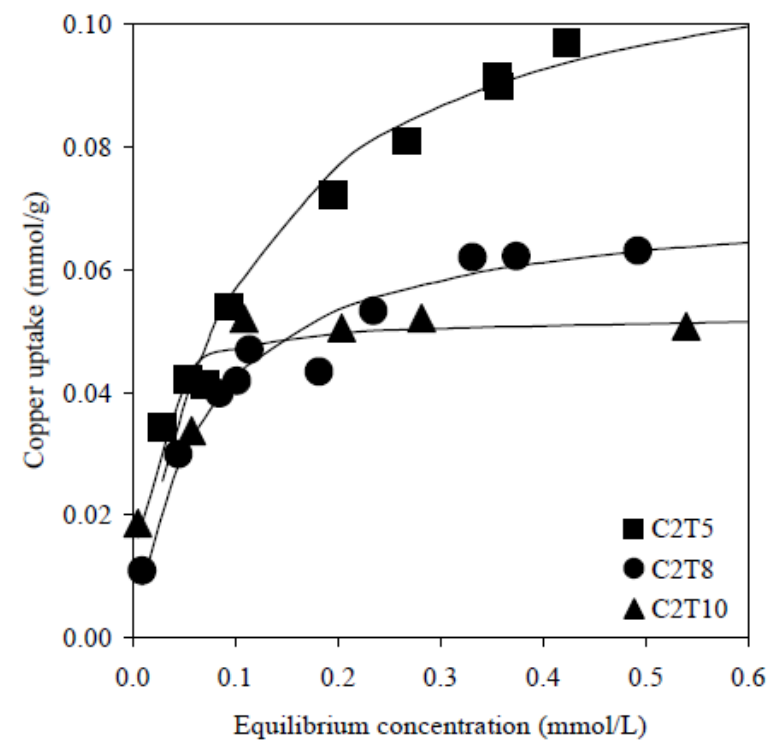

Figure 2. Adsorption of copper ions by C2 activated carbons series. Lines were predicted from Langmuir model.

localized active sites, and is given by,

$$
q_{e}=\frac{C_{e} \cdot Q \cdot b}{1+b \cdot C_{e}}
$$

where $Q$ and $b$ represent maximum uptake and affinity, respectively. The Freundlich model describes multilayer adsorption on a heterogeneous surface, and is given by,

$$
q_{e}=K_{F} \cdot C_{e}^{\frac{1}{n}}
$$

where $K_{F}$ and $n$ are Freundlich constants related to the uptake capacity and intensity, respectively. Parameters of 
isotherm models and coefficients of determination $\left(r^{2}\right)$ are tabulated in Table 3.

For both activated carbons series, the adsorption data can be satisfactorily described, and were more fitted to linear approximation by Langmuir model. The values of maximum uptake, $Q$, were well agreed with the plotted adsorption data in Figures $\mathbf{1}$ and $\mathbf{2}$.

When acidic groups that correspond to electron withdrawers are removed during helium degassing at higher temperature, the $\pi$-system will become rich with electron density and thus operative for metal ions adsorption through $\mathrm{C} \pi$-cation interaction. This could also contribute to the increase of affinity towards copper ions, regardless the decrease in surface area. The trend of affinity given by $\mathrm{C} 2$ series was more prevalent as compared to $\mathrm{C} 1 \mathrm{se}-$ ries because of the mesopore-rich nature. It is suggested that copper ions favor to dehydrate on the mesopores at lower equilibrium concentration.

Figure 3 illustrates the mechanisms of copper ions dehydration onto the surface CMC-activated carbons. It is inferred that the adsorption of copper ions takes place on mesopore channels $(2-50 \mathrm{~nm})$ at lower equilibrium concentration. As the surface approaching saturation at higher equilibrium concentration, the copper ions move to micropore channels $(<2 \mathrm{~nm})$.

Table 3. Parameters of Isotherm Models.

\begin{tabular}{ccccccc}
\hline \multirow{2}{*}{ Carbon } & \multicolumn{3}{c}{ Langmuir model } & \multicolumn{4}{c}{ Freundlich model } \\
\cline { 2 - 7 } & $\boldsymbol{Q}$ (mmol/g) & $\boldsymbol{b}(\mathbf{L} / \mathbf{m m o l})$ & $\boldsymbol{r}^{2}$ & $\boldsymbol{K}_{\boldsymbol{F}}$ & $\mathbf{1} \boldsymbol{n}$ & $\boldsymbol{r}^{2}$ \\
\hline C1T5 & 0.060 & 6.87 & 0.99 & 0.061 & 0.42 & 0.95 \\
C1T8 & 0.055 & 11.9 & 0.98 & 0.070 & 0.41 & 0.97 \\
C1T10 & 0.056 & 29.1 & 1.00 & 0.096 & 0.42 & 0.96 \\
C2T5 & 0.117 & 9.50 & 0.99 & 0.138 & 0.41 & 0.99 \\
C2T8 & 0.072 & 14.3 & 0.98 & 0.100 & 0.43 & 0.94 \\
C2T10 & 0.052 & 83.6 & 1.00 & 0.070 & 0.24 & 0.89 \\
\hline
\end{tabular}

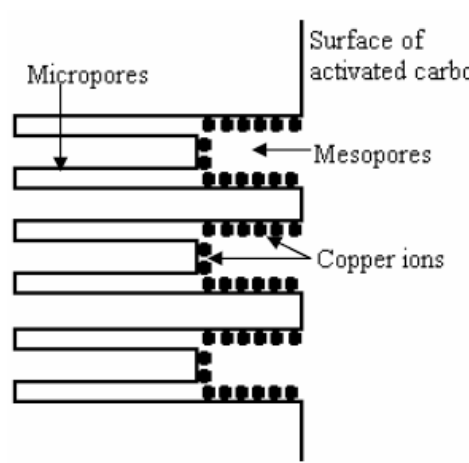

(a)

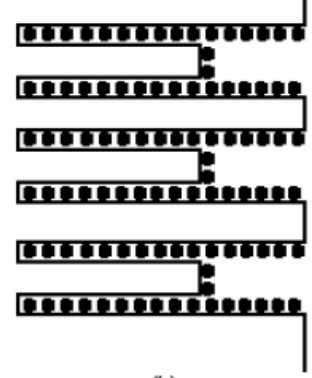

(b)
Figure 3. Schematic representation of dehydration of copper ions onto CMC-activated carbos. (a): at lower equilibrium concentration; (b): at higher equilibrium concentration. (Note: Not to scale).

\section{Conclusions}

Conclusions that can be drawn form this work are listed as follows.

- Helium degassing at higher temperature has resulted in the increase of surface basic nature, and the decrease of surface area of CMC-activated carbons.

- Adsorption of copper ions was mainly driven by surface area and electron-rich $\pi$-system of graphene layers. Significant decrease in surface area reduced the uptake of copper ions.

- Adsorption data were well correlated by Langmuir model, i.e. monolayer dehydration of copper ions onto the surface of CMC-activated carbons.

- The increase of affinity towards copper ions at lower equilibrium concentration could be attributed to the increase of electron density on graphene layers, regardless the decrease in surface area.

- At lower equilibrium concentration, copper ions were preferably dehydrated on mesopores, and thereafter switched to micropores as the surface approaching saturation.

\section{Acknowledgements}

This work was part of M.A.A. Zaini doctoral thesis at Chiba University. It was funded in part by the Japan Society for the Promotion of Science under Grants-in-aid for Scientific Research (C) (No. 20510072). M.A.A. Zaini thanks Ministry of Higher Education, Malaysia and Universiti Teknologi Malaysia (UTM) for the SLAI-financial sponsorship. Travel bursary by Centre of Lipids Engineering \& Applied Research (CLEAR) is greatly appreciated.

\section{REFERENCES}

[1] WHO, "Guidelines for Drinking Water Quality," World Health Organization, Geneva, 2006.

[2] T. A. Kurniawan, G. Y. S. Chan, W.-H. Lo and S. Babel, "Physico-Chemical Treatment Techniques for Wastewater Laden with Heavy Metals," Chemical Engineering Journal, Vol. 118, No. 1-2, 2006, pp. 83-98. doi:10.1016/j.cej.2006.01.015

[3] R. C. Bansal and M. Goyal, "Activated Carbon Adsorption," CRC Press, Boca Raton, 2005. doi:10.1201/9781420028812

[4] Roskill, "The Economics of Activated Carbon," Roskill Information Services Ltd., London, 2008.

[5] J. M. Dias, M. C. M. Alvim-Ferraz, M. F. Almeida, J. Rivera-Utrilla and M. Sánchez-Polo, "Waste Materials for Activated Carbon Preparation and Its Use in AqueousPhase Treatment: A Review," Journal of Environmental Management, Vol. 85, No. 4, 2007, pp. 833-846. doi:10.1016/j.jenvman.2007.07.031

[6] Q. Qian, M. Machida and H. Tatsumoto, "Preparation of 
Activated Carbons from Cattle-Manure Compost by Zinc Chloride Activation," Bioresource Technology, Vol. 98, No. 2, 2007, pp. 353-360. doi:10.1016/j.biortech.2005.12.023

[7] Q. Qian, Q. Chen, M. Machida, H. Tatsumoto, K. Mochidzuki and A. Sakoda, "Removal of Organic Contaminants from Aqueous Solution by Cattle Manure Compost (CMC) Derived Activated Carbons," Applied Surface Science, Vol. 255, No. 12, 2009, pp. 6107-6114. doi:10.1016/j.apsusc.2009.01.060

[8] M. A. A. Zaini, R. Okayama and M. Machida, "Adsorption of Aqueous Metal Ions on Cattle-Manure-Compost-
Based Activated Carbons," Journal of Hazardous Materials, Vol. 170, No. 2-3, 2009, pp. 1119-1124. doi:10.1016/j.jhazmat.2009.05.090

[9] H. P. Boehm, "Some Aspects of the surface Chemistry of Carbon Blacks and Other Carbons," Carbon, Vol. 32, No. 5, 1994, pp. 759-769. doi:10.1016/0008-6223(94)90031-0

[10] I. D. Smiciklas, S. K. Milonjic, P. Pfendt and S. Raicevic, "The Point of Zero Charge and Sorption of Cadmium (II) and Strontium (II) Ions on Synthetic Hydroxyapatite," Separation and Purification Technology, Vol. 18, No. 3, 2000, pp. 185-194. doi:10.1016/S1383-5866(99)00066-0 